\title{
DU MONDE QUI NE VA PAS SANS DIRE
}

\author{
Élise MARROU
}

Sorbonne Université, Faculté de lettres

C'est le langage qui fait du réel un monde. Cette thèse ne charrie-t-elle pas quelques relents d'idéalisme - un idéalisme qui mettrait seulement le langage à la place des idées, ou de la conscience, et dont le mot d'ordre serait: «Tenons-nous en au langage, c'est la seule réalité - du moins la seule que nous puissions atteindre ?» Nullement. Car la thèse ne prétend pas que «tout est langage», ou que le réel se « réduit» au langage. Elle dit même tout le contraire.

Elle pose que le langage fait du réel un monde.

Francis Wolff, Dire le monde

Que faut-il pour faire un monde? À cette question classique, Francis Wolff répondait en $1997 \mathrm{ni}$ de tout (car le monde n'est pas une collection de choses, une totalité entendue au sens purement quantitatif ou extensionnel d'un inventaire exhaustif), ni seulement du tout (car le monde ne répond pas non plus à la seule exigence d'universalité ou d'universalisation de l'expérience), mais « des noms, des verbes et des pronoms personnels ${ }^{1} \gg$. Sa réponse avait de quoi surprendre le lecteur. Elle garde toute sa dimension provocatrice aujourd'hui. À suivre les linéaments de la démonstration de l'ouvrage, le langage « fait monde » en un double sens : la prédication donne accès au monde; cet usage apophantique du logos est par définition intersubjectif. C'est donc le langage qui fait du réel un monde entendu à la fois comme tout ordonné et comme réel partagé. Il y aurait dès lors un cercle vertueux entre la relation d'objectivité et la relation d'interlocution, ainsi qu'une réversibilité de principe entre le langage défini comme objet-monde et la structure elle-même langagière du monde. On ne saurait distinguer le monde de ce que nous pouvons en dire. Par définition, le monde ne peut donc aller sans dire, ni même aller de soi.

Ce sont précisément les modalités de cette circularité que nous nous proposons de discuter ici. Nous souhaiterions le faire en nous souvenant que les premiers ouvrages que nous avons lus de Francis Wolff portaient sur Aristote et que les cours auxquels

\footnotetext{
${ }^{1}$ Dans ce qui suit, nous nous référons à la seconde édition de Dire le monde (désormais DM), Paris, Presses uuniversitaires de France, coll. « Quadrige », 2004, p. 5.
} 
nous avons d'abord assisté à l'École étaient ses cours d'agrégation sur le Stagirite. Un trait commun s'en dégageait - ou plutôt une double insatisfaction s'y exprimait : une impatience conceptuelle et une volonté d'en découdre avec une manière paresseuse et stérile de faire de l'histoire de la philosophie. En effet, de Logique de l'élément à Penser avec les Anciens, Francis Wolff a défendu et mis en pratique une méthode qu'il qualifie lui-même de « $\operatorname{mixte}^{2} »:$ l'historien de la philosophie ne renonce en rien à la dimension problématisante des questions qu'il affronte; le philosophe se défait, quant à lui, du mythe du commencement absolu en héritant de figures de pensées qu'il s'approprie, mais dont il reste en dernière instance tributaire :

Il se peut que nous ne puissions penser que dans des formes héritées. Mais cela ne signifie pas que nous devions nous contenter de recueillir l'héritage. En pensant avec la philosophie ancienne, il doit être possible de philosopher aujourd'hui. Emprunter aux Anciens, c'est leur prendre ce qui ne laisse pas de leur appartenir, c'est donc tenter de les lire fidèlement en accommodant notre regard historique sur eux, mais c'est aussi à l'inverse tenter de les comprendre complètement en assimilant leur pensée à la nôtre. C'est s'efforcer de sortir de l'alternative : histoire ou philosophie ${ }^{3}$.

Ce cercle vertueux de l'inscription historique et du questionnement conceptuel peut être décrit de bien des manières et pratiqué sans doute selon des modes encore plus divers. Dans chacun de ses ouvrages, Francis Wolff maintient pourtant l'exigence d'affronter les questions philosophiques pour ce qu'elles sont, sans en réduire la profondeur ni la problématicité, en leur apportant des réponses aussi tranchantes qu'elles sont limpides; de là dans son œuvre, la pratique constante de la définition. Il est hors de doute que Francis Wolff est lui aussi un « définisseur hors pair $»^{4}-$ encore faut-il s'entendre sur sa manière singulière d'hériter d'Aristote et d'en retraduire les leçons. C'est ce que nous nous proposons de faire ici : nous partirons d'une exposition des thèses et de la démarche d'ensemble de Dire le monde, avant d'en extraire deux points nodaux qui nous paraissent pertinents à titre de contre-épreuve, voire de pierre de touche, pour prolonger la discussion.

Revenons donc sur les premières pages de l'essai. Il ne s'ouvre ni sur une thèse ni sur une définition, mais sur ce que nous nommerons faute de mieux une clause d'indissociabilité : loin de décrire l'ameublement du monde, Francis Wolff répond à une toute autre question: «que doit-être le monde pour être dicible?». Cette

${ }^{2}$ Logique de l'élément. Clinamen, Paris, Puf, 1981, p. 12.

${ }^{3}$ Penser avec les Anciens, Paris, Fayard, coll. « Pluriel », 2016, p. 7.

${ }^{4}$ Francis Wolff qualifie Aristote de «définisseur hors pair » (L'Être, l’homme, le disciple, Paris, Puf, coll. « Quadrige », 2000, p. 103), le compliment est ici transitif. 
interrogation marque le déplacement de la métaphysique entendue dans le sens le plus traditionnel d'une discipline qui s'interroge sur la totalité et qui pose la question «pourquoi ? » à une position que l'auteur nomme lui-même « critique ». La méthode est donc transcendantale, mais en un autre sens que celui d'une constitution du monde par l'aperception de l'ego. Notre finitude contresigne notre incapacité à saisir les choses mêmes, nous ne pouvons que nous contenter $d u$ monde pour nous :

Du monde « en soi », on ne peut rien dire, mais le monde «pour nous » est tout ce qu'on peut en dire $^{5}$.

Dès lors, la question de l'antécédence ou de la priorité de l'un sur l'autre ne saurait jamais être tranchée. Francis Wolff maintient toutefois l'isomorphisme du langage et du monde, sans que le langage et le monde ne soient selon lui deux ensembles liés de manière bijective. C'est précisément de cette tentation, ou de cette entente de l'isomorphisme, que l'ouvrage nous prévient ${ }^{6}$. L'intuition originaire qui en est la source est que nous ne pouvons précisément pas adopter une perspective extérieure au monde ni au langage de les saisir pour les relier, ni même à partir de laquelle nous serions en mesure d'appréhender que l'un est le miroir de l'autre. L'un et l'autre sont totalisants. L'un et l'autre sont réfléchissants. Nous y sommes plongés. Aussi Francis Wolff peut-il affirmer avec une pointe d'ironie humienne qu' « il serait absurde de prétendre que notre langage prescrit des lois aux choses; il serait fantastique qu'il y ait une harmonie préétablie entre les lois du monde en soi et les lois de notre langage ${ }^{7}$ ». Le sens de la question (que faut-il pour faire un monde?) en sort radicalement transformé : Francis Wolff pose ici une question de droit, elle ne concerne donc pas les langues telles que nous les utilisons couramment ou le monde tel que nous l'habitons, mais « les conditions pour que nous puissions parler du monde ${ }^{8} 》$. On passe ainsi des questions classiques et traditionnelles «Qu'y a-t-il au monde ou dans le monde ? » « ou «Qu'est-ce qui constitue le monde ? » à celle, plus précise, à laquelle l'ouvrage s'attaque frontalement : «Que doit être le monde pour que nous puissions le dire ?». Après avoir formulé les enjeux de cette clause d'indissociabilité, nous dégagerons deux axes de discussion : le premier (1) porte sur le concept soustractif du réel qui en résulte; le second (2) sur le statut de la parole-monde.

${ }^{5}$ DM, p. 5.

${ }^{6} \mathrm{Ce}$ en quoi, contrairement à ce que son auteur affirme (DM, p. 11), Dire le monde réitère ici la décision qui avait guidé Wittgenstein dans le Tractatus.

${ }^{7}$ DM, p. 56.

${ }^{8}$ DM, p. 31. 
(1) À admettre en effet que la thèse de l'antécédence du monde sur le langage ou celle de l'accès au monde indépendamment de toute ressource langagière est dépourvue de sens, c'est-à-dire à adopter une position que Francis Wolff nomme lui-même « critique », le réel ne saurait par définition se confondre avec le sens soustractif que l'essai lui attribue pourtant. Cette détermination privative du réel n'est-elle pas ellemême qu'un produit dérivé ou un succédané de l'illusion dont la démarche critique de l'auteur entendait nous prémunir (celle d'un point de vue surplombant ou angélique à partir duquel nous serions en mesure de saisir le monde, mais également l'écart séparant le monde pour nous du réel en soi) ?

(2) À maintenir que « le monde est tout ce qu'on peut en dire, mais que nul ne peut tout dire du monde », n'est-on pas contraint de rectifier la définition initiale du monde comme « tout ce qui est et là où tous nous sommes »? Dès lors la parole-monde ne défait-elle pas et ne refait-elle le monde en y agissant (tout autant qu'elle le fait)?

\section{Le déplacement initial de la question : que faut-il pour faire un monde?}

Au-delà de la provocation évidente, la pertinence de la question qui ouvrait l'ouvrage de 1997 tenait d'abord au déplacement imposé à chacun de ses termes. Tout d'abord, par « faire » monde, Francis Wolff n'entendait pas plus une fabrique (poieisis) qu'une opération de constitution. Dire que les noms, les verbes et les pronoms personnels font monde, ce n'est donc pas dire qu'ils sont les briques qui le construisent, en un mot, que le monde serait leur œuvre ou leur édification. Ce n'est pas non plus soutenir que l'expérience mondaine résulte de l'activité constituante d'un ego transcendantal, fût-il parlant. En soutenant que « le langage fait monde », Francis Wolff défendait que l'on ne saurait distinguer le monde de ce que nous pouvons en dire :

Selon la position critique, la forme du langage, c'est l'ordre du monde ; il est donc aussi impossible qu'inutile de tenter de séparer ce qui revient à l'un et à l'autre 9 .

Cette décision initiale renvoyait ainsi dos à dos le réalisme métaphysique réalisme qu'on pourra nommer alternativement naï, robuste ou naturel qui tient que nous avons un accès non problématique au réel en soi - et une grammaticalisation de l'expérience qui réduirait le monde à l'opération de nos catégories linguistiques. Par le refus assumé de choisir entre un monde à décrire et un monde à construire, Francis Wolff récusait également que nous fussions acculés à trancher de manière unilatérale

${ }^{9}$ DM, p. 31. 
entre les deux projets concurrents de la phénoménologie husserlienne et du constructionnisme de Carnap. Et il le faisait en formulant une hypothèse historique reprise dans Penser avec les Anciens :

La pensée contemporaine a sans doute délaissé l'interrogation directe sur le monde, mais tout se passe comme si, pour continuer à philosopher, c'est-à-dire à interroger le réel comme un tout, il lui avait fallu le faire de manière oblique en choisissant un « objetmonde» qui puisse s'y substituer. Ces objets sont divers selon les courants. $\mathrm{Vu}$ d'aujourd'hui où la tendance dominante consiste à réinterpréter l'histoire de la philosophie du $\mathrm{XX}^{\mathrm{e}}$ siècle comme ayant été partagée en deux courants principaux, la phénoménologie et la philosophie analytique, ces deux voies de la philosophie que tout oppose paraissent avoir un point essentiel en commun. Elles interrogent non le monde luimême, mais reflété ou déjà donné dans une structure qui en tient lieu et qui a les mêmes propriétés que lui, la conscience ou le langage, l'un et l'autre sont des structures de coappartenance totalisantes, réfléchissantes et qui se présentent comme donnant accès immédiatement au monde. Ce dernier point est sans doute problématique. Chacun de ces objets-mondes pourrait contester à l'autre sa prétendue immédiateté et lui dénier toute donation des choses mêmes: du point de vue de la conscience, le langage apparaît comme une médiation superflue ou pire un miroir déformant. À quoi le langage pourrait légitimement répondre que ce qu'il perd en immédiateté de l'expérience, il le gagne en richesse. Le monde qui s'offre à la conscience, on y est d'emblée, mais on y est seul : l'intersubjectivité ou l'objectivité n'en sont pas de données immédiates. Le langage surcharge sans doute le réel de formes, de structures et de règles qui ne s'y trouvent pas $a$ priori, mais le monde ainsi parlé est d'emblée un monde commun. En somme, conscience et langage apparaissent comme deux rivaux qui prétendent donner le monde à sa place ${ }^{10}$.

Ces deux objets-mondes n'opèrent toutefois pas de manière symétrique. Francis Wolff précise en effet que le langage fait monde d'une manière singulière qui n'est pas celle de la conscience. Car totalisante et réfléchissante, la conscience l'est également. Si le langage reste toutefois un objet-monde privilégié, c'est que la relation qui l'unit au monde est selon l'auteur plus « intime» que celle qui unit la conscience au monde. C'est le langage qui fait du réel un monde en l'ordonnant et en le rendant commun :

Le monde, c'est le réel appréhendé comme un tout ordonné, c'est aussi le réel mis en commun $^{11}$.

Dire, c'est d'une part nécessairement dire le monde, car dire, c'est dire quelque chose à propos de quelque chose (legein ti kata tinos), dire c'est prédiquer. On touche ici le cœur de la thèse de l'ouvrage. Dire le monde, c'est au fond revenir au sens premier de la prédication :

\footnotetext{
${ }^{10}$ Penser avec les Anciens, « Les deux destins de l'ontologie », op. cit., p. 66-67.

${ }^{11}$ DM, p. 6.
} 
Pour que le langage soit possible, il faut poser la distinction entre ce dont on parle et ce qu'on en dit. Cette distinction prise au sens très large du « sujet» et du "prédicat » est sans doute un universel du langage. Il faut que des interlocuteurs puissent parler de la même chose (le « sujet») - en la posant implicitement comme la même qu'elle-même et donc comme n'étant pas contradictoire - et en dire l'un et l'autre autre chose (que ce sujet est tel ou non tel). Sans la première condition, on ne pourrait parler de rien; sans la seconde, on ne pourrait rien dire. Sans la première condition, rien n'accrocherait du réel qu'un discours puisse saisir ; sans la seconde, tout serait déjà dit et il n'y aurait rien à dire. $^{12}$

D'où les deux règles dialogiques formulées par l'auteur; les deux conditions du dialogue (parler de la même chose/ pouvoir en dire autre chose). À chacune de ces normes linguistiques répond une exigence ontologique : l'unité de l'essence (toujours identique jamais contradictoire) et la multiplicité indéfinie des accidents (autres, au point de pouvoir être contradictoires). En outre, ce dire n'est pas solitaire, puisqu'en disant quelque chose du monde, j'inclus nécessairement, fût-ce virtuellement, un interlocuteur dans mon dire. Francis Wolff est ici tout à fait fidèle à la Rhétorique d'Aristote et cette fidélité est l'un des leitmotive de ses ouvrages :

Trois éléments constitutifs sont à distinguer pour tout discours : celui qui parle, ce dont il parle, celui à qui il parle ${ }^{13}$.

Le fait que le logos ne permet pas de penser l'objectivité comme un problème n'est ici que l'envers du fait que le logos associe toujours objectivité et interlocutivité. Le monde est ainsi « une structure totale d'entités objectives et un milieu commun à tous ${ }^{14} »$. Nos mots ne font pas écran au monde sauf à comprendre qu'ils le révèlent. Il n'y a pas d'un côté, les mots, et de l'autre, les choses, opposition donc terme à terme dont l'un des risques pourrait être que les mots rendraient les choses opaques ou notre relation au monde lui-même opaque. Au contraire, les noms, les verbes et les pronoms personnels sont, pour reprendre une image employée dans le livre, « la fenêtre même du monde ». Le langage est transparent ou encore «nous sommes dans le langage comme dans une cage transparente ${ }^{15} »$. Si la relation entre le langage et le monde est plus intime que ne l'est celle de la conscience et du monde, ce n'est donc pas parce que l'articulation de la proposition mimerait ou serait le calque de la structure du monde, c'est en raison même de l'impossibilité de nous extraire de l'un et de l'autre pour juger

${ }^{12}$ DM, p. 52.

${ }^{13}$ Aristote, Rhétorique, I, 3, 1358a37-38.

14 DM, p. 6.

${ }^{15}$ DM, p. 26. 
de l'une indépendamment de l'autre; dans le dire que Francis Wolff nomme un langage-monde, noms et verbes sont liés dans une proposition ou une série des propositions dont la visée et la vocation sont essentiellement descriptives. Mais « il y a plus », comme l'écrit souvent notre auteur: nos mots en tant qu'ils peuvent être également les vecteurs d'une parole-monde, et non plus d'un langage-monde, renvoient à la situation d'un agent, d'une personne dont le monde n'est plus seulement un habitacle ou un habitat, mais vecteur de l'action. Le monde dont le sens n'était jusqu'à présent que cosmologique (il était littéralement cosmologique) prend sens. Deux modalités de notre rapport au monde se trouvent ainsi dégagées au moyen de deux nouveaux vocables, « le langage-monde » par lequel le monde est décrit, « la parolemonde » par laquelle nous y trouvons notre place par notre action.

Pour mieux comprendre cette progression, partons du langage-monde :

Le monde est pour nous fait de choses identiques à elles-mêmes et d'événements qui leur arrivent, parce qu'il faut des sujets nommables et des prédicats qu'on puisse leur attribuer $^{16}$.

Cette conclusion procède des deux expériences de pensée, de deux «critiquesfiction » qui sont l'un des tours de force de l'essai qui consiste à renvoyer dos à dos deux mondes fictifs : un monde de l'identité pure et un monde de l'événementialité pure. Ces deux fictions sont la pierre de touche qui permet de dégager les limites du monde, ou encore la structure de notre monde. Dans la première, le langage-monde de l'identité pure, c'est un monde d'essences qui est supposé, dans le langage qui dit ce monde de l'identité pure un mot a un sens si et seulement s'il se réfère à une seule chose; la distinction entre désigner et attribuer n'a plus lieu d'être, parler, c'est ici nommer. À l'inverse, dans la seconde, celle du monde de la différence pure, un monde où tout ne serait accident, dans ce monde tout change, sans qu'aucun substrat fixe ne demeure. Le langage n'est plus nominal, mais verbal. Les questions « qu'est-ce que ?» et la question «pourquoi ? » en sont les traces : que doit-être le monde pour que ces deux questions prennent sens? D'abord, un monde d'individus à propos desquels on peut demander ce qu'ils sont (la condition en est le principe de non-contradiction). Ensuite, un lien entre ces individus qui rende compte de l'événementialité du monde (la condition demeure le principe de raison). Nous n'avons pas à déplorer de ne pas vivre dans le premier monde, celui de l'identité. Nous ne devons pas non plus regretter de ne pas vivre dans le second monde, celui de l'événementialité pure. Notre finitude nous

${ }^{16}$ DM, p. 149. 
empêche certes de saisir les choses mêmes, mais si on consent à la retraduire positivement, ces signes de finitude sont les conditions même de ce que doit être le monde pour que nous puissions le dire :

Il faut à la fois que les choses soient ce qu'elles sont, comme dans le monde des choses, pour qu'on en puisse parler, et qu'elles ne soient jamais tout à fait ni seulement cela mais toujours aussi autre chose pour qu'on en puisse dire quelque chose ${ }^{17}$.

Nous pouvons réaffirmer ce que nous disions en commençant :

Le monde est pour nous est fait de choses identiques à elles-mêmes et d'événements qui leur arrivent, parce qu'il faut des sujets nommables et des prédicats qu'on puisse leur attribuer $^{18}$.

Envisageons enfin la troisième et dernière vague, la parole-monde dont on aurait tort de sous-estimer l'importance : par la première, le langage fait monde au sens où nous le disons, où nous le racontons ou nous le rapportons ; par la seconde, nous faisons le monde au sens où nous y agissons. Jusqu'à présent, Francis Wolff n'avait envisagé que le devenir de la sumplokè du Sophiste, l'entrelacs, le tissage des noms et des verbes reformulés dans les termes aristotéliciens de l'attribution prédicative. Il s'agit désormais à partir de la parole-monde de se demander « qui agit ?». On ne saurait répondre à la question de l'imputation en gardant un concept exclusivement métaphysique de la personne : il s'agit bien plutôt de rendre compte de l'agent, c'est-à-dire littéralement de la personne qui agit et qui ne peut se réduire à un support d'actes, à un suppôt que l'on pourrait décrire en troisième personne. La question transcendantale est donc reprise et reformulée : «que doit-être ce monde où existent les personnes et leurs actes pour que nous puissions en parler ? $^{19} »$. À cette question, la réponse de la position critique n'est plus la personne au sens métaphysique du terme, mais la personne au sens grammatical. Insistons sur ce point : c'est la personne entendue en un sens grammatical qui lève, pour reprendre les termes de Francis Wolff, «l'antinomie de la chose et de l'événement ${ }^{20} »$. L'auteur inscrit ses analyses ici dans la continuité de celles de Benveniste (« est ego qui dit ego »), de Jean-Claude Pariente et d'Ernst Tugendhat. Alors que le langage-monde se signalait par sa transparence, la parole-monde - pourrait-on suggérer - se distingue par sa résistance, ou du moins par l'insistance sur sa position dans le monde : «Le

\footnotetext{
${ }^{17}$ DM, p. 109.

${ }^{18}$ DM, p. 149.

${ }^{19}$ DM, p. 120.

${ }^{20}$ DM, p. 156.
} 
monde n'est plus tout ce dont on peut parler, mais tous ceux qui peuvent en $\operatorname{parler}^{21}$ ». À l'impératif catégorique, Francis Wolff substitue comme forme première de l'éthique ce qu'il nomme «l'interrogatif éthique » qui est toujours problématique et donc jamais apodictique : « est-ce que par le fait de demander « qui agit ? », je consens à m'inscrire dans le monde comme agent ? ». Cette question précède elle-même l'obéissance à la loi morale, car c'est à la seule condition d'être dans un monde où j'existe d'abord en tant qu'agent que je peux avoir accès à un véritable universel, plutôt qu'à ses faux semblants, et éviter de me conduire pour reprendre l'expression d'Hannah Arendt citée par Francis Wolff comme un « petit homme».

\section{Deux points de discussion :}

\section{(a) Un concept soustractif de réel}

Si nous prenons à présent quelque recul après avoir observé la déferlante de ces trois vagues successives exposées dans Dire le monde, ordre de la nomination, ordre de la prédication, ordre de la réflexivité, il est clair que la position critique défendue par Francis Wolff résulte de la conjonction d'une inspiration aristotélicienne, issue, de l'aveu de l'auteur, du livre Gamma de la Métaphysique, et d'un héritage kantien proche des Individus de Strawson. Si nous évoquons cette conjonction, c'est parce qu'elle nous paraît délicate à tenir jusqu'au bout et pour tout dire instable. Ce n'est donc pas tant la circularité assumée de la démarche qui nous paraît poser ici problème (elle peut tout à fait être vertueuse et féconde) que la rencontre de ces deux voies que nous avons nommées, donc pour le dire vite, aristotélicienne et critique. Nous voudrions suggérer en nous souvenant des enseignements de Francis Wolff sur Aristote qu'il pourrait s'en tenir à la première source d'inspiration, sans l'inscrire pour autant dans un cadre kantien $^{22}$.

Empruntons donc la première voie aristotélicienne : revenant sur l'établissement du principe de non-contradiction comme principe des principes en Gamma $^{23}$, Francis Wolff rappelle que pour Aristote le principe de non-contradiction n'est pas une règle de

${ }^{21}$ DM, p. 186

${ }^{22}$ La mise en place de Dire le monde est profondément liée à ce que dans Après la finitude, Quentin Meillassoux a nommé «corrélationnisme». Voir Après la finitude. Essai sur la nécessité de la contingence, Seuil, 2012, chapitre 1.

${ }^{23}$ Comme Francis Wolff le précise, on peut lire Dire le monde dans son ensemble comme une réflexion sur les conditions de possibilité du principe de non-contradiction. "Deux destins de l'ontologie » développe d'une manière approfondie la confrontation entre ce principe aristotélicien et le Cogito cartésien. 
grammaire, il ne régit pas seulement la proposition ; il n'est pas formel, il porte sur les êtres eux-mêmes. C'est de l'aveu même de l'auteur la source même d'où provient Dire le monde: pour reprendre les termes plus littéraux d'Aristote, celui qui refuse d'accepter le principe de non-contradiction s'excepte de l'ordre du monde et de la communauté des hommes. Le logos n'est pour Aristote tout d'abord rien d'extérieur aux phénomènes qu'il décrit ou qu'il explique. Sa vertu descriptive ou explicative s'inscrit dans le prolongement de nos sens. À tout phénomène étaient associées pour les Grecs les dimensions d'une intelligibilité discursive. En outre - Francis Wolff est l'un de ceux qui ont sans doute le mieux établi l'importance de cette distinction aristotélicienne - la différence qui sépare le logos de la phonè réside pour Aristote dans le fait même qu'il communique non des affects mais des valeurs :

À la vertu sémantique de la voix, le langage proprement humain ajoute la vertu politique de désigner (dêloun) les valeurs communes et de les mettre en commun ${ }^{24}$.

Il paraît ainsi nécessaire de doubler le logos entendu comme faculté d'exprimer et de communiquer par concepts cette thèse profondément aristotélicienne selon laquelle l'homme vit dans un milieu où il peut percevoir ce qui constitue l'objet même du politique, c'est-à-dire les valeurs sociales. Or, c'est précisément la richesse de ce modèle à la fois aphophantique et social que l'on risque de perdre si l'on retraduit l'idiome aristotélicien dans celui de Strawson pour les délester de leur lourdeur métaphysique ${ }^{25}$.

Gagnons-nous quoi que ce soit à retraduire ainsi la position d'Aristote dans les termes d'une « ontologie critique qui n'atteint que les êtres qui nous apparaissent pour que nous puissions en $\operatorname{parler}^{26} »$ ? Il nous semble que nous y perdons d'autant plus que Francis Wolff a lui-même profondément réaménagé et déplacé le projet de métaphysique descriptive de Strawson pour ne garder que ce que son épure a de plus audacieux. C'est le premier point qui offre matière à notre objection. Accordons tout d'abord sans difficulté un point sur lequel nous ne reviendrons pas par la suite: l'objection d'idéalisme est déjouée ; compte tenu des prémisses de l'analyse de Francis

\footnotetext{
${ }^{24}$ Aristote et la politique, Paris, Puf, coll. « Philosophies », 1991, p. 74. Le lecteur pourra également se reporter à « L'homme politique entre Dieu et bête », dans Penser avec les Anciens, p. 145-147.

${ }^{25}$ Nous pensons ici tout particulièrement aux pages 56-58 de Dire le monde où Aristote en prend pour son grade.

${ }^{26}$ DM, p. 5.
} 
Wolff, elle ne peut effectivement même pas être formulée ${ }^{27}$. Nous avons vu dans la première étape de cette contribution que la force de la position critique tenait avant tout à ce que nous avons nommé la clause d'indissociabilité : nous avons admis sans difficulté que la thèse de l'antécédence du monde sur le langage ou celle de l'accès au monde indépendamment de toute ressource langagière était dépourvue de sens. Mais il faut toutefois s'entendre sur ses conséquences. L'une d'entre elles qui nous paraît problématique tient à la distinction entre le monde et le réel. Le passage ci-après la cristallise et c'est pourquoi nous citerons Dire le monde longuement :

Le langage fait monde - comme la conscience. Mais il y a peut-être entre le langage et le monde une relation encore plus intime. Et qui voudrait, par exemple, caractériser à grands traits la manière dont le monde nous apparaît, trouverait peut-être dans la manière dont il transparaît dans le langage la voie la plus sûre. Car c'est bien au monde qu'il a affaire et pas seulement au « réel ». Qu'est-ce que le monde sans le langage? Tout ce qu'on en peut percevoir. Et par percevoir, il ne faut pas entendre seulement recevoir et mémoriser passivement des sensations, à la manière d'un appareil enregistreur, mais aussi, comme l'animal, explorer activement les sinuosités de la réalité, se heurter à ses contours, expérimenter sa résistance, éprouver ses agréments et ses tourments. Le réel ainsi perçu n'est pas un pur chaos : il n'est pas simplement doté d'une «matière » et de qualités (couleurs, sons, odeurs, plaisirs, douleurs, désirs, craintes, images, souvenirs ...), mais il est aussi, sans doute, doté de «formes » et structuré par des percepts. Chaque champ perceptif a sa propre organisation interne (l'étendue visuelle, ou la durée auditive par exemple), comme le montrent les travaux de psychologie ou les recherches sur la «cognition». Mais il ne semble pas que cet ordre intrinsèque quelles que soient sa complexité et sa portée, soit du même niveau que celui imposé par le langage. C'est pourquoi le problème demeure de savoir comment les concepts peuvent dériver de percepts ou s'articuler à eux, et surtout comment l'ordre propre à chaque champ perceptif peut d'une part s'harmoniser avec celui des autres champs en un ensemble cohérent (c'est-à-dire non seulement agencé par des mécanismes régulateurs mais bien pensable comme une réalité unique) et d'autre part, s'accorder avec celui des autres sujets percevants en une expérience objective à propos de laquelle tous puissent penser de concert. En somme, la forme et la matière du perçu sont locales et propres, alors que la syntaxe et la sémantique du langage sont générales et communes. Le perçu ne « fait » pas monde parce qu'il lui manque et la coappartenance de toutes choses à une même structure d'ensemble (le renvoi de tout donné réel à un possible jamais donné, la cohérence des parties, la connexion des séquences) et l'extériorité objective structurée en concepts par la mise en commun de l'expérience ${ }^{28}$.

\footnotetext{
${ }^{27}$ Pour un développement de ce point, le lecteur pourra se reporter à sa contribution « De l'inévitabilité rationnelle de l'idéalisme à la nécessité logique du réalisme» dans le volume Choses en soi. Métaphysiques du réalisme, E. Alloa et E. During (éd.), Puf, coll. « Philosophie », 2018, p. 185-200.

${ }^{28}$ DM, p. 26-27.
} 
Il ne s'agit évidemment pas d'objecter à Francis Wolff que Dire le monde aurait dû comporter en son sein une théorie de la perception en bonne et due forme ou une forme de conceptualisme moins abruptement formulé, mais de suggérer plutôt que deux choses l'une :

(1) soit l'on adopte une position critique au sens kantien du terme. Dans ce cas, à supposer que nous l'adoptions jusqu'au bout, nous n'avons pas plus accès au réel via la perception que par nos concepts. La position critique est clairement corrélationniste et s'associe à un diagnostic de finitude dont on peut discuter le bien-fondé, mais dont il est difficile de contester que la perception ne saurait nous en extirper. Le réel ne saurait dès lors être défini de manière littéralement soustractive comme « le monde sans langage ». Or c'est exactement la définition du réel proposée par Francis Wolff dans l'extrait cidessus. À adopter ce criticisme réaménagé, on perd le double bénéfice du pacte apophantique hérité d'Aristote : l'inscription du logos dans le prolongement de nos sens et sa dimension sociale.

Cette perte nous semble d'autant plus préjudiciable qu'on comprend mal les raisons qui autoriserait la « position critique » à pouvoir confronter le monde pour nous auquel le langage nous permet d'accéder et le réel auquel la perception nous donnerait directement accès, alors même que Francis Wolff souligne l'ordre intrinsèque de chaque champ perceptif ou sensoriel. Cette détermination privative du réel apparaît donc comme un produit dérivé de l'illusion dont la démarche critique de l'auteur entendait nous prémunir (celle d'un point de vue surplombant ou angélique à partir duquel nous serions en mesure de saisir le monde), car ce n'est qu'à condition d'adopter ce point de vue surplombant que nous pourrions faire la part du monde pour nous et celle du réel en soi. Or, c'est impossible pour les raisons « critiques » déjà exposées par l'auteur. Poser ainsi que le perçu ne fait pas monde, mais que nous y avons d'emblée accès par nos sens nous paraît constituer une retombée tout à fait classique dans le mythe du donné alors même que l'ouverture de l'ouvrage pouvait être lue comme une tentative de le désamorcer : la circularité d'emblée assumée nous paraît prometteuse dans la mesure exacte où elle refuse à la fois de souscrire à la thèse d'un monde antéprédicatif dont le dire ne serait qu'une trahison foncière et d'un monde entièrement constitué par nos catégories linguistiques. L'objection rejaillit de plus belle si l'on se souvient que la relation d'interlocutivité qui fait du réel un monde commun et partagé doublait le sens du monde cosmologique d'un sens phénoménologique au sens large d'habitation, d'occupation de ce monde que Francis Wolff nommait « milieu ». À prendre l'auteur au mot, la relation d'interlocutivité faisait-elle de notre monde (Welt) également un 
milieu (Umwelt). La dimension axiologique de l'analyse d'Aristote pouvait ici reprendre ses droits et être intégrée à l'analyse du monde.

(2) soit on maintient la force de la position aristotélicienne initiale et on assume pleinement ce qui est établi en Gamma, l'articulation forte entre la déterminité du sens et le fait que le langage porte sur les choses mêmes. On peut sans doute aménager l'intuition réaliste d'Aristote et le faire dans la direction indiquée par Putnam. Selon Hilary Putnam, le réalisme métaphysique nécessite l'adoption d'un «point de vue divin », nécessairement illusoire parce qu'il l'est de croire que nous pouvons accéder à un monde tout à fait indépendant des conditions particulières (sociales, psychologiques ou culturelles) de sa représentation. Cette voie nous paraît féconde pour asseoir et consolider le réalisme des Anciens et le monde tout court, mais il faut alors renoncer au cadre critique dans lequel Francis Wolff a inscrit sa réflexion.

\section{(b) Une totalité ouverte?}

Francis Wolff conclut Dire le monde par cette formule : « le monde est tout ce qu'on peut en dire, mais nul ne peut tout dire du monde». N'est-on pas dès lors contraint de rectifier la définition initiale du monde comme « tout ce qui est et là où tous nous sommes»? L'auteur souligne avec force par cette formule la dimension d'ouverture et d'inachèvement du monde lui-même, mais l'essai semble ne pas en tirer toutes les conséquences. Nous avons vu, en présentant rapidement les linéaments de la parole-monde, que cet inachèvement était profondément lié à ce que Francis Wolff nomme lui-même « l'interrogatif éthique ». Mais il n'est pas seulement lié à la première personne du singulier, mais à la première personne du pluriel comprise dans sa dimension et son évolution historiques. Certes, nous nous accordons tout à fait avec l'auteur sur les dangers de l'historicisme soulignés dans la préface de l'essai. Si le monde est par définition un monde commun ou un réel partagé, encore faut-il qu'il y ait partage précisément et que le partage puisse avoir lieu. Comment formuler la clause d'appartenance si ce n'est dans un «nous »? C'est tout alors à la fois l'esprit commun et le sentiment d'appartenance au corps politique, l'ensemble des normes tacites qui constituent le socle de vivre-ensemble, la condition de la communauté. Cette parole commune garde une part d'événementialité et d'opacité qui en est constitutive. C'est ce qu'indique le statut du «nous » qui n'apparaît qu'en pointillés dans l'essai de 1997 comme sujet. C'était déjà le sens de la réponse de Pierre Macherey en 2001 et que ce dernier retraduisait pour sa part dans des termes spinozistes : 
En parcourant l'itinéraire qui conduit du langage-monde, avec ses fallacieuses promesses, à la parole-monde qui, ayant abandonné ses sécurités, est d'une certaine façon une parole de détresse, à la manière de celle adressée par celui que le silence des espaces infinis effraie, et qui s'est convaincue que nulle lumière ne peut tirer Dieu de l'obscurité où il s'est définitivement retiré et caché, mais aussi à la manière de celui qui a renoncé aux illusions de la métaphysique, on se donne les moyens de dire et de faire le monde autrement, c'est-à-dire aussi de lui reconnaître et de lui donner une véritable histoire. Le monde que dit la parole-monde est proprement, et à tous les sens du mot, un monde sans fin, qu'on n'aura jamais fini historiquement de dire et de faire, car les perspectives sous lesquelles on l'aborde doivent rester pour toujours ouvertes, sans qu'aucun avenir radieux puisse venir en combler ou en refermer le ciel. Désillusionné, désenchanté, on s'engage dans ce monde en sachant qu'on n'en fera jamais le tour, même en y employant beaucoup plus que quatre-vingt jours : mais c'est justement pour cela qu'on s'y engage, au plein sens du terme, donc à ses propres frais, avec pour seule conviction que, c'est la dernière phrase de Dire le monde, « si le monde est tout ce qu'on peut en dire, nul ne peut tout dire du monde $»^{29}$.

Faire monde en ce dernier sens, c'est avoir la possibilité de défaire le monde présent, déjà ancien et de le refaire. Cette seconde direction de discussion n'est pas tant une objection qu'une volonté de poursuivre l'horizon même de l'essai en suggérant pour conclure que ce n'est peut-être pas tant d'une remontée aux conditions de possibilité de tout discours qu'il était ici fondamentalement question, mais des éventuels risques qu'il y aurait à distinguer de manière étanche l'ontologie qu'Aristote nous a transmise de l'ancrage politique qui était le sien. N'est-ce pas là la leçon profonde de Gamma? N'est-ce pas celle-là même dont Francis Wolff nous a appris à hériter et dont son œuvre est la plus éclatante illustration ${ }^{30}$ ? Pour cette même raison, entre le réel et le monde, il faut sans aucun doute distinguer, mais faut-il choisir ${ }^{31}$ ? Nous avons tenté de donner ici quelques pistes qui permettent d'en douter.

\footnotetext{
${ }^{29}$ Cette recension de Pierre Macherey parue en 2001 dans les Études philosophiques est désormais accessible en ligne ici : https://www.cairn.info/revue-les-etudes-philosophiques-2001-1-page-109.htm

${ }^{30}$ Le lecteur pourra par exemple se reporter à la confrontation entre raison réflexive et raison dialogique par laquelle « Les deux voies de la métaphysique » se termine, L'Être, l'homme, le disciple, op. cit, p. 96102 ainsi qu'aux analyses subtiles et profondes menées dans « L'homme politique entre dieu et bête », art. cit., p .139-155.

${ }^{31}$ Voir les conclusions de l'essai «Les deux destins de l'ontologie », dans L'Être, l'homme, le disciple, op. cit., tout particulièrement p. 68.
} 


\section{Bibliographie}

Aristote, Rhétorique, introduction, traduction, notes, bibliographie et index par P. Chiron, Flammarion, coll. GF, 2007.

Macherey Pierre, compte-rendu de F. Wolff Dire le monde dans Les Études philosophiques, 2001/1 (56), p. 109-116. url : https://www.cairn.info/revue-les-etudes-philosophiques-2001-1page-109.htm

Meillassoux Quentin, Après la finitude. Essai sur la nécessité de la contingence, Seuil, 2012.

Wolff Francis, Logique de l'élément. Clinamen, Paris, Presses universitaires de France, 1981.

Wolff Francis, Aristote et la politique, Paris, Presses universitaires de France, coll. « Philosophies », 1991.

Wolff Francis, L'Être, l'homme, le disciple, Paris, Presses universitaires de France, coll. «Quadrige », 2000.

Wolff Francis, Dire le monde, Paris, Presses universitaires de France, coll. « Quadrige », 2004.

Wolff Francis, Penser avec les Anciens, Paris, Fayard, coll. « Pluriel », 2016.

Wolff Francis, «De l'inévitabilité rationnelle de l'idéalisme à la nécessité logique du réalisme », dans E. During et E. Alloa (éd.), Choses en soi. Métaphysiques du réalisme, Presses universitaires de France, coll. « Philosophie », 2018, p. 185-200. 